\title{
A MapX-based Segmentation Algorithm of Region Feature by Polyline
}

\author{
http://dx.doi.org/10.3991/ijoe.v9iS4.2658 \\ Da-Sheng $\mathrm{Wu}^{1,2}$ \\ 1Zhejiang University, Hangzhou, China, ${ }^{2}$ Zhejiang A \& F University, Lin'an, Zhejiang, China
}

\begin{abstract}
MapX didn't provide a ready function which can be easy to split region feature in the client. The objective of this study was to design a segmentation algorithm for cutting region feature with polyline. In order to convenience the description, during the segmentation, only 2 intersection points were taken into consideration. According to the order of P1(the first intersection point) and P2(the second intersection point) in the $R$ (region feature) and $L$ (polyline feature), 4 kinds of situations had been taken into account, those were respectively, $P 2$ was always after $P 1$ in the $R$ and $L, P 2$ was after $P 1$ in the $R$ but $P 2$ was before $P 1$ in the $L$, $P 2$ was always before $P 1$ in the $R$ and $L$, and $P 2$ was before $P 1$ in the $R$ but $P 2$ was after P1 in the $L$. Segmentation results showed that the algorithm was stable and reliable.
\end{abstract}

Index Terms-MapX, Forest resource, Region feature, Segmentation algorithm.

\section{INTRODUCTION}

GIS(Geographic information system) had been more widely used to forest resources management along with the continuous development of "3S" (RS, GIS, GPS) and other relational hardware and software technologies[1-5]. To some extent, as MapInfo, accepted by many users because of its simplicity and ease of use with more comprehensive functionality[6-7]. Of course, no matter what type of GIS software, considering the functional versatility, for general users, its operation was still too complex, which provided a market space for developing specialized geographic information system by programming with object-oriented programming language and GIS middleware.

Sub-compartment was the basic unit for forest resource management, and its area should be adjusted according to different management objective. Usually, in forest resources GIS, a sub-compartment should be represented as a region feature and its size should be adjusted by splitting or merging. The region feature, stored and displayed as a form of vector data, was composed of a series of ordered points, and each point was represented by one pair of coordinates $(\mathrm{x}, \mathrm{y})$. Specifically, the first point and the last point was the same point in region feature.

MapX was a kind of middleware technology that can provide most of the function of MapInfo, and it was frequently used in programming for specialized GIS[811]. But it did not provide a ready feature segmentation algorithm which could be easy to split dynamically subcompartment in the client. Considering that the boundary of the region feature was usually composed by polyline with a lot of points, although previous study had described segmentation algorithm about region feature divided by straight-line[12], it should be improved.
To solve this problem, this paper designed a MapXbased segmentation algorithm of region feature by polyline, and it was programmed by VB6.0.

\section{DESCRIPTION OF THE PROBlem}

Supposing that R should be segmented into R1 and R2, Pts1 and Pts2 were the ordered point sets about R1 and R2 respectively, $\mathrm{L}$ was a polyline, $\mathrm{L}$ and $\mathrm{R}$ intersected in 2 points of $\mathrm{P} 1$ and $\mathrm{P} 2$ whose coordinates were P1.X and $\mathrm{P} 1 . \mathrm{Y}$ in $\mathrm{P} 1$, and $\mathrm{P} 2 . \mathrm{X}$ and $\mathrm{P} 2 . \mathrm{Y}$ in $\mathrm{P} 2$, meanwhile, $\mathrm{P} 1 . \mathrm{X}$ $<=\mathrm{P} 2 . \mathrm{X}$

According to the order of $\mathrm{P} 1$ and $\mathrm{P} 2$ in the $\mathrm{R}$ and $\mathrm{L}$, generally there were 4 segmentation as following

(1) As shown in Fig.1(a), P2 was always after P1 in the $\mathrm{R}$ and L. Where, Pts1=\{1,P1,2',3',4',P2,3,4,5 $\}$ and Pts2 $=\left\{\mathrm{P} 1,2, \mathrm{P}^{2}, 4^{\prime}, 3^{\prime}, 2^{\prime}\right\}$.

(2) As shown in Fig.1(b), P2 was after P1 in the R, but P2 was before $\mathrm{P} 1$ in the $\mathrm{L}$. Where, Pts $1=\left\{1, \mathrm{P} 1,4^{\prime}, 3^{\prime}, 2^{\prime}, \mathrm{P} 2,3,4,5\right\} \quad$ and $\mathrm{Pts} 2=\{\mathrm{P} 1,2, \mathrm{P} 2$, 2',3',4'\}

(3) As shown in Fig.1(c), P2 was always before P1 in the $\mathrm{R}$ and $\mathrm{L}$. Where, Pts1 $=\left\{1, \mathrm{P} 2,2^{\prime}, 3^{\prime}, 4^{\prime}, \mathrm{P} 1\right\}$ and Pts2 $=\left\{\mathrm{P} 2,2,3,4,5, \mathrm{P} 1,4^{\prime}, 3^{\prime}, 2^{\prime}\right\}$

(4) As shown in Fig.1(d), P2 was before P1 in the R, but $\mathrm{P} 2$ was after $\mathrm{P} 1$ in the $\mathrm{L}$. Where, Pts1 $=\{1, \mathrm{P} 2$, 4',3',2',P1 $\}$ and Pts2= P2,2,3,4,5, P1,2',3',4' $\}$

In particular, when the $\mathrm{P} 1$ or $\mathrm{P} 2$ coincides with a vertex of $\mathrm{R}$, just only one of them should be added to the Pts1 and Pts2.

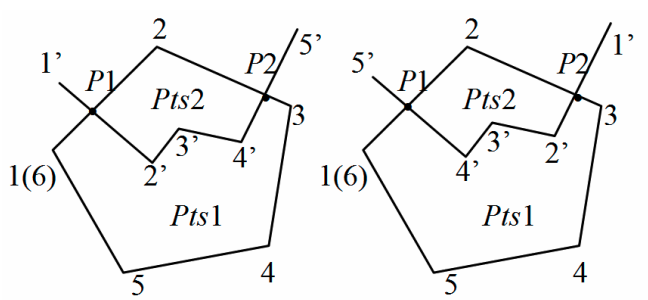

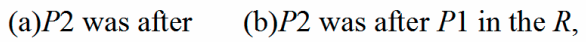
$P 1$ in the $R$ and $L$ but $P 2$ was before $P 1$ in the $L$

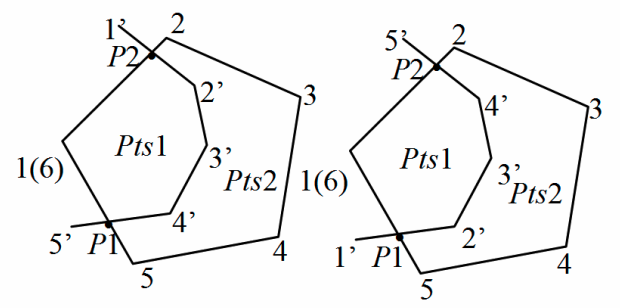

(c) $P 2$ was before (d) $P 2$ was before $P 1$ in the $R$, $P 1$ in the $R$ and $L$ but $P 2$ was after $P 1$ in the $L$

Figure 1. The 4 kinds of segmentation 


\section{A MapX-BAsed Segmentation Algorithm of Region Feature by Polyline}

\section{AlgORITHM DESIGN}

Now supposing that there were $n$ vertices, and $n-1$ edges on the region feature of $R$, and $E j(1 \leqslant j \leqslant n-1)$ was an edge which had 2 endpoint of $\mathrm{Pj}$ and $\mathrm{Pj}+1, \mathrm{Pj}$ indicated the jth vertex; ptsChange was a boolean variable whose value could decide the points of $\mathrm{R}$ and $\mathrm{L}$ to be added to Pts1 or Pts2.

(1)Set ptsChange to false

(2)Looped for each edge of Ej, and the value of $\mathrm{j}$ was from 1 to $n-1$. Where, a virtual rectangular should be created which took Ej as main diagonal or sub-diagonal, $\operatorname{minX}$ and $\operatorname{maxY}$ as the left-top corner coordinates, $\operatorname{maxX}$ and $\operatorname{minY}$ as the right-bottom corner coordinates correspondingly. The P1 was located in Ej if and only if $\min X \leqslant P 1 . X \leqslant \max X$ and $\min Y \leqslant P 1 . Y \leqslant \max Y$, that meant the location of P1 in R was found. Similarly, it should be found that the position of the point of $\mathrm{P} 2$ located in $\mathrm{R}$ also.

(3)Similarly, they could be found that the position of the points of $\mathrm{P} 1$ and $\mathrm{P} 2$ located in $\mathrm{L}$.

(4)Obtained the point sets of Pts1 and Pts2, and their process detailed in the program flow diagram (shown in Fig.2).

(5)Converted the point sets of Pts1 and Pts2 into independent region features which should be added into current layer immediately.

(6)Delete the polyline feature of $\mathrm{L}$ and the region feature of $\mathrm{R}$.

\section{ALGORITHM IMPLEMENTATION}

The following algorithm was based on the programming environment of Visual Basic 6.0 and MapX5.0.

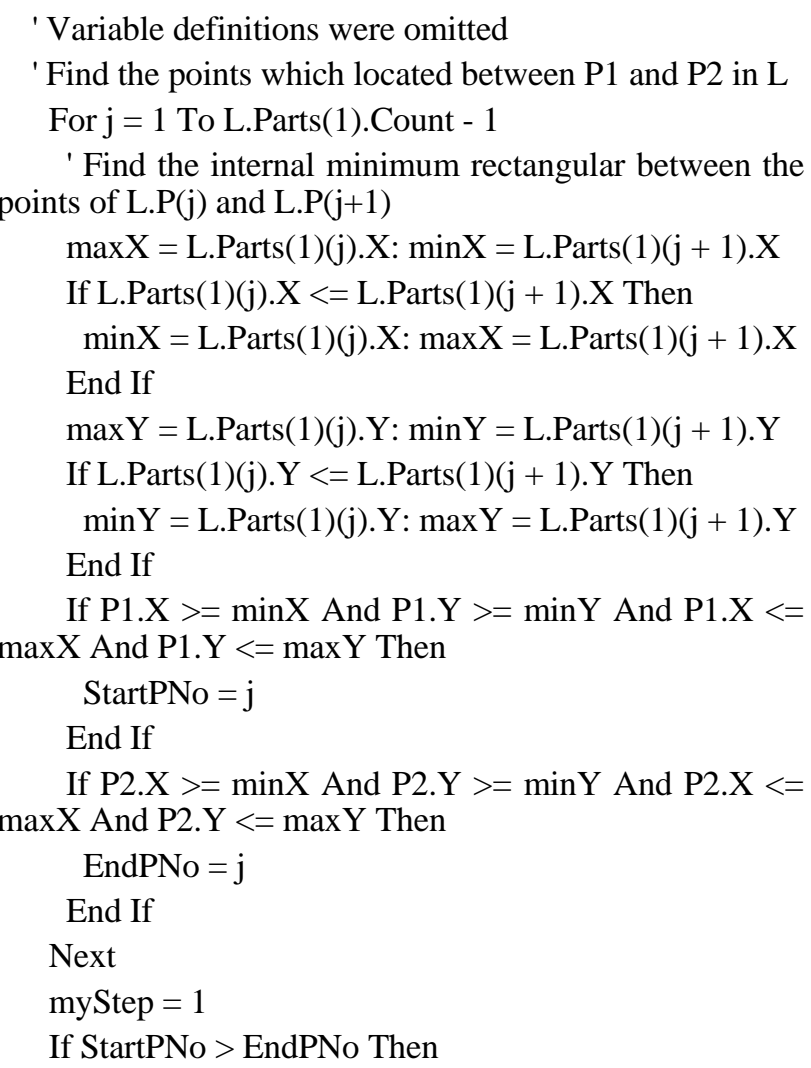

myStep $=-1$

EndPNo = L.Parts(1).Count - EndPNo: StartPNo = L.Parts(1).Count - StartPNo +1

Else

StartPNo $=$ StartPNo +1

End If

P2afterP1 $=0$

For j = 1 To R.Parts(1).Count - 1

' Find the internal minimum rectangular between the points of R.P(j) and R.P(j+1), and codes are skipped

' Process the common points

If Not $((P 1 . X=$ R.Parts(1)(j).X And P1.Y = R.Parts(1)(j).Y) Or (P2.X = R.Parts(1)(j).X And P2.Y = R.Parts(1)(j).Y)) Then ' When the intersection points do not coincide with the points of R.P(j)

If Not ptsChange Then

Pts1.Add R.Parts(1)(j)

Else

Pts2.Add R.Parts(1)(j)

End If

End If

' Process the intersection point of P1

If $\mathrm{P} 1 . \mathrm{X}>=\min \mathrm{X}$ And $\mathrm{P} 1 . \mathrm{Y}>=\min \mathrm{Y}$ And $\mathrm{P} 1 . \mathrm{X}<=$ $\max X$ And $\mathrm{P} 1 . \mathrm{Y}<=\max \mathrm{Y}$ Then

Pts1.Add P1: Pts2.Add P1: P2afterP1=P2afterP1+ 1

If myStep=1 Then 'when the $\mathrm{P} 1$ is before $\mathrm{P} 2$ in $\mathrm{L}$

If P2afterP1=1 Then 'when the P1 is before P2 in R

For $\mathrm{k}=$ StartPNo To EndPNo Step myStep

Pts1.Add L.Parts(1)(k) ' add the points to Pts1

Next

Else ' when the $\mathrm{P} 1$ was after $\mathrm{P} 2$ in the $\mathrm{L}$

For $\mathrm{k}=$ StartPNo To EndPNo Step myStep

Pts2.Add L.Parts(1)(k) ' add the points to Pts2

Next

End If

Else ' when the P1 was after P2 in the $\mathrm{R}$

If P2afterP1 = 1 Then

For $\mathrm{k}=$ EndPNo To StartPNo Step myStep

Pts1.Add L.Parts(1)(k) ' add the points to Pts1

Next

Else

For $\mathrm{k}=$ EndPNo To StartPNo Step myStep

Pts2.Add L.Parts(1)(k) ' add the points to Pts2

Next

End If

End If

If P1.X = R.Parts(1)(j).X And P1.Y = R.Parts(1)(j).Y Then 'when P1=R.P(j)

$$
\mathrm{j}=\mathrm{j}+1
$$

End If

ptsChange $=$ Not ptsChange

End If

' Considering its processing similarity to the intersection point of $\mathrm{P} 1$, the process of $\mathrm{P} 2$, its codes is also skipped. 
Next

' Process the last point of $\mathrm{R}$

If lastPoint $=\mathrm{P} 1$ Or lastPoint $=\mathrm{P} 2$ Then

Pts1.Add R.Parts(1)(j): Pts2.Add R.Parts(1)(j) Else

If Not ptsChange Then

Pts1.Add R.Parts(1)(j)

Else

Pts2.Add R.Parts(1)(j)

End If

End If

Set R1 = FtrFac.CreateRegion(Pts1, R.Style.Clone): Set R2 = FtrFac.CreateRegion(Pts2, R.Style.Clone)

Set ftrnew1 = lyr.AddFeature(R1): Set ftrnew2 = lyr.AddFeature(R2) "add the R1 and R2 into the layer of lyr

lyr.DeleteFeature R: lyr.DeleteFeature ' delete the old features of $\mathrm{R}$ and $\mathrm{L}$

\section{THE SIMULATION RESULTS}

As shown in Fig.3, the simulation results indicated that the algorithm had achieved to the segmentation to region feature based on polyline.

\section{CONCLUSION}

MapX did not provide a ready segmentation algorithm which could be easy to split region feature in the client dynamically. To solve this problem, this paper proposed a MapX-based segmentation algorithm of region feature by polyline. The algorithm had taken a variety of complex segmentation into account based on 2 intersection points. Segmentation results showed that the algorithm was stable and reliable.

\section{ACKNOWLEDGMENT}

This work was financially supported by Zhejiang province major science and technology project (2011C12047) and Zhejiang province education department project(Y201225717).

\section{REFERENCES}

[1] Chu, L.X., Zhang, L., "Design of forest resources data system based on GIS database", Journal of Central South University of Forestry \& Technology, vol.32, no.6, pp.48-54, 2012.
[2] Wu, L.C., Li, C.G., Chen, H.A., "Design and implementation of the universal forestry resource survey GIS”, Science of Surveying and Mapping, vol.36, no.4, pp.255-257, 2011.

[3] Li, X.D., Zhang, Z., "The development of GIS-based archive management system of forest farm resources", Journal of Changchun University, vol.20, no.12, pp. 69-73, 2010.

[4] Singh, I.J., Jugran, D.K., Thanruma, S., Reddy, S.R., "Forest resource assessment in mohand forest range, Uttar Pradesh using remote sensing and GIS”, Journal of the Indian Society of Remote Sensing, vol.33, no.4, pp.565-574, 2005. http://dx.doi.org/10.1007/ $\underline{B F 02990741}$

[5] McCall, M.K., Minang, P.A., “Assessing participatory GIS for community-based natural resource management: Claiming community forests in Cameroon”, Geographical Journal, vol.171, no.4, pp.340-356, 2005. http://dx.doi.org/10.1111/j.1475-4959. 2005.00173.X

[6] Huang, S.C., Xu K.J., "Establishment and Application of GIS of Forest Resources at County Level Based on Mapinfo", Journal of Guangxi Academy of Sciences, vol.22 no.4, pp.333-336, 2006.

[7] Xiao, W., "Forest Distribution Mapping of Hubei Province based on MapInfo Software", Forest Inventory and Planning, vol.31, no.2, pp.17-20, 2006.

[8] Zhang, Q., Yin, X., "Best path analysis in military highway transportation based on MapX”, Advances in Intelligent and Soft Computing, vol.129, pp. 163-168, 2011. http://dx.doi.org/10.1007/ 978-3-642-25986-9_25

[9] Liu, X.D., Chen, Y. W., Long, Y.J., “A MapX-based preprocessing approach for multi-satellite cooperative observation towards area target”, System Engineering Theory and Practice, vol.30, no.12, pp.2269-2275, 2010.

[10] Wu, J., Niu, D., Liu, S., "Design and implementation of rainfall retrieval system of weather radar based on MapX”, Journal of Geomatics, vol.35, no.3, pp.19-20, 2010.

[11] Yan, P., Wu, W., Wang, X., "Pilot design of biomass energy resources inquiry system based on MapX: A case of Liyang city in China”, International Journal of Global Energy Issues, vol.29, no.3, pp.294-302, 2008. http://dx.doi.org/10.1504/IJGEI.2008.0 $\underline{18010}$

[12] Wu, D.S., Feng, X., Wen, Q.Q., “The segmentation algorithm of region feature based on MAPX”, Advances in Intelligent and Soft Computing 105(2011) 657-661. http://dx.doi.org/10.1007/978-3642-23756-0_105

\section{AUTHORS}

Da-Sheng Wu is with Institution of Remote Sensing and Information System Application, Zhejiang University, Hangzhou 310029, China, Tel: 1-396-802-3105, (E-mail: wu62390710@263.net).

This article is an extended and modified version of a paper presented at the International Conference on Mechanical Engineering, Automation and Material Science (MEAMS2012), held 22-23 December 2012, Wuhan, China. Received 08 April 2013. Published as resubmitted by the authors 01 May 2013. 


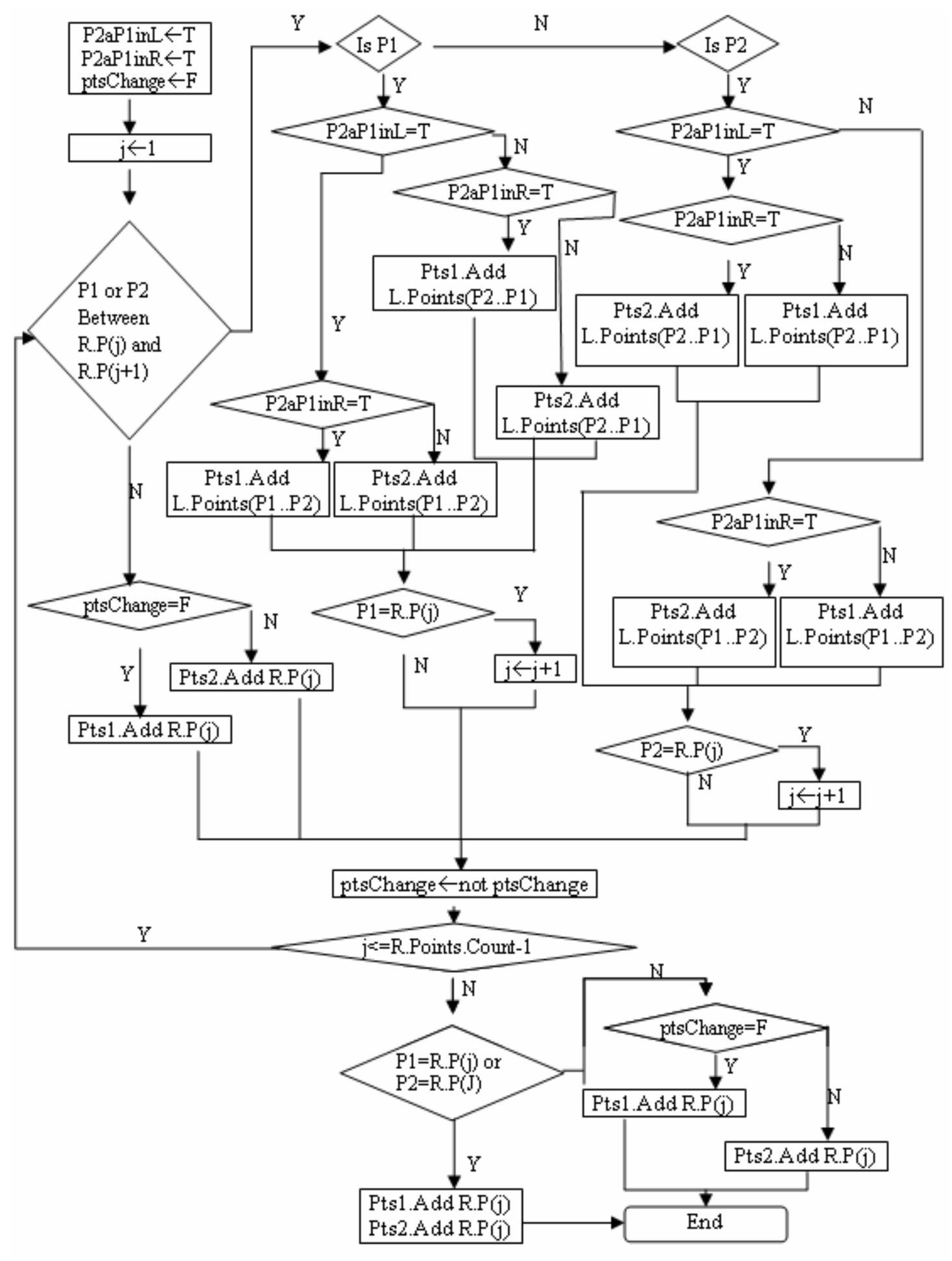



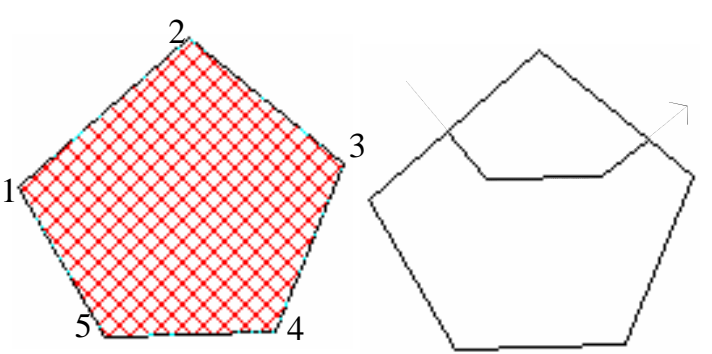

(a) select the feature of $R 1$

(b) $R 1$ segmented by $L 1$ when $P 2$

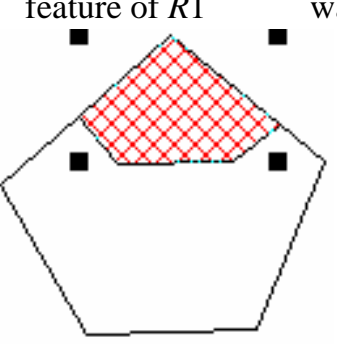

was after $P 1$ in the $R 1$ and $L 1$

(c)select the feature of $R 2$

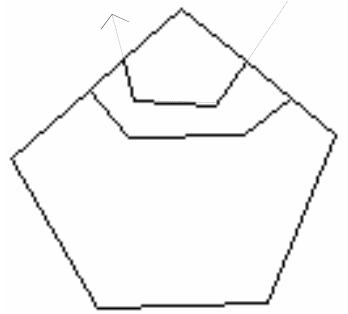

(d)R2 segmented by $L 2$ when $P 2$ was after $P 1$ in the $R 2$ but before $P 1$ in the $L 2$
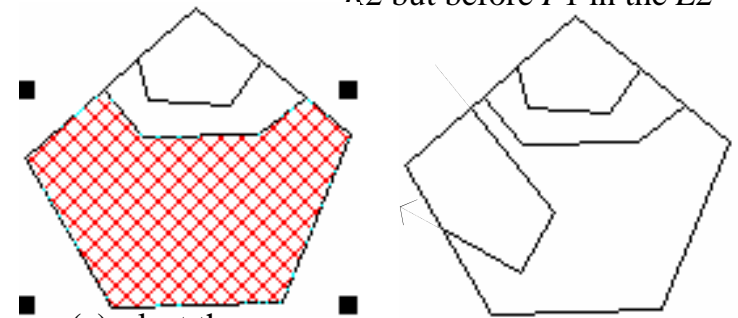

(e)select the feature of $R 3$

(f) $R 3$ segmented by $L 3$ when $P 2$
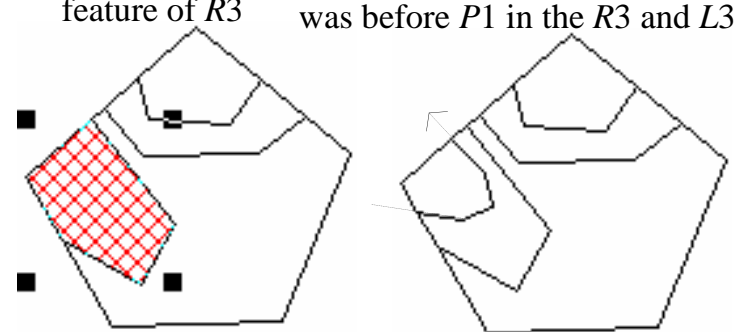

(h)R4 segmented by $L 4$ when $P 2$

(g)select the

Feature of $R 4$ was before $P 1$ in the $R 4$ but was after $P 1$ in the $L 4$
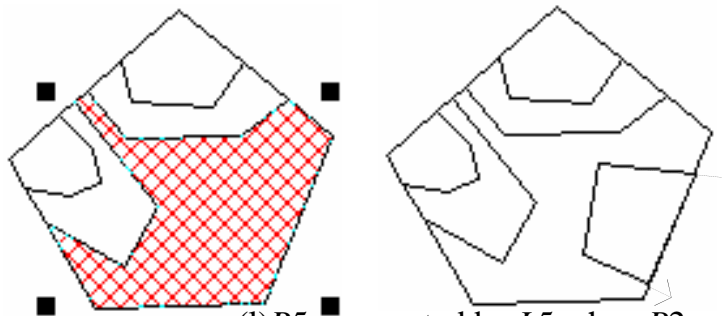

(l) $R 5$ segmented by $L 5$ when $P 2$ was

(k)select the before $P 1$ in the $R 5$ and $L 5$, meanwhile $P 2$ feature of $R 5$ and $P 1$ were in the same eage in the $R 5$
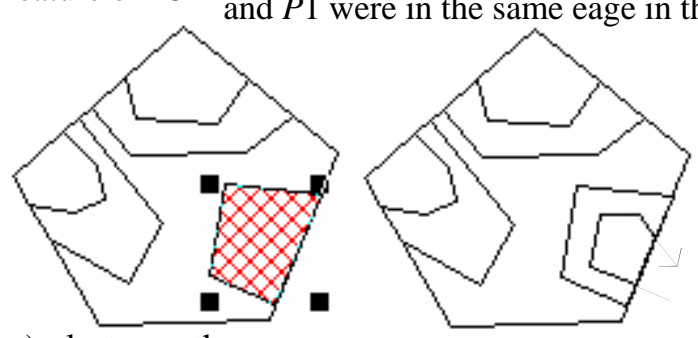

(m)select feature of $R 6$

(n) $R 6$ segmented by $L 6$ when $P 2$ was before $P 1$ in the $R 6$ but after $P 1$ in the $L 6$, meanwhile $P 2$ and $P 1$ were in the same eage in the $R 6$

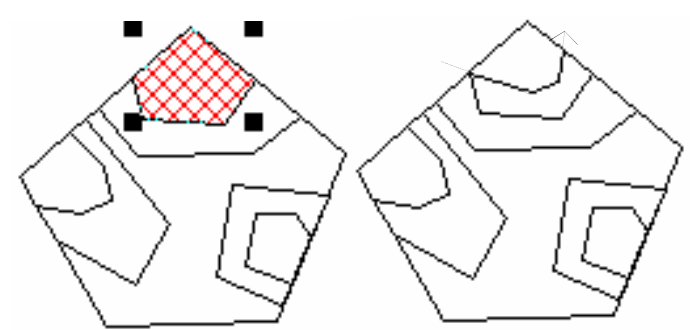

(m)select the

(n)R7 segmented by $L 7$ when $P 2$ feature of $R 7$ was after $P 1$ in the $R 7$ and $L 7$, meanwhile $P 1$ coincided with a vertex of the $R 7$

Figure 3. The Result of Segmentation 